\title{
Synthesis, crystal growth, thermal and spectroscopic studies of acentric materials constructed from aminopyridines and 4-nitrophenol
}

\author{
Ilia M. Pavlovetc, ${ }^{1}$ Sergiu Draguta, ${ }^{2}$ Maria I. Fokina, ${ }^{1}$ Tatiana V. Timofeeva, ${ }^{2}$ and Igor Yu.Denisyuk ${ }^{1 *}$ \\ ${ }^{1}$ Department of Engineering Photonics, ITMO University, St. Petersburg 197101, Russia. \\ Email:*denisiuk@mail.ifmo.ru \\ ${ }^{2}$ Department of Biology and Chemistry, New Mexico Highlands University, Las Vegas, NM 87701, USA
}

\begin{abstract}
A series of single co-crystals of non-centrosymmetric complexes of different mono- and diaminopyridines, namely 4-aminopyridine, 3,4-diaminopyridine, 2-amino-6-methylpyridine, 2,6-diaminopyridine, 2-aminopyridine, with 4-nitrophenol were grown by slow evaporation technique from solution at constant temperature. Optical transparency in the region of 300-1100 nm was found to be suitable for nonlinear optical applications with cut off wavelengths at $420-474 \mathrm{~nm}$ for these co-crystals. Single crystal X-ray analysis confirms the non-centrosymmetric packing. Thermogravimetric and differential thermal analysis shows good thermal stability of the studied compounds with melting point at range $99-172^{\circ} \mathrm{C}$ for different co-crystals. The second harmonic generation efficiency has been measured on single crystals by comparative SHG technique relative to KTP crystal. The nonlinear optical coefficient of the compounds was found to be up to $39 \mathrm{pm} / \mathrm{V}$.
\end{abstract}

Keyword: nonlinear optical materials, second harmonic generation, molecular crystals, optical nonlinearities in organic materials, thermal analysis.

\section{Introduction}

During last years, organic nonlinear optical materials with high second-order nonlinear susceptibilities has found some applications in electro-optical devices such as optical waveguides or frequency modulators [1]. Three classes of electro-optical (EO) materials are used today: inorganic crystals (e.g., $\mathrm{LiNbO}_{3}$ ), polymers or dendrimers doped with electro-optical dye molecules, and organic crystals with large EO coefficients [2,3]. Among organic crystals mostly well known is 4-N,N-dimethylamino-4'-N'-methylstilbazolium tosylate (DAST), especially interesting material for nonlinear optical (NLO) device applications due to its large EO coefficient and a low dielectric constant $(\varepsilon=5.2)$, giving rise to a high modulator figure of merit and thermal stability up to $250^{\circ} \mathrm{C}$ [4]. According to data in [5], DAST exhibits the second-order NLO susceptibility $\chi^{(2)}=2020 \pm 220 \mathrm{pm} / \mathrm{V}$ at $\lambda=1318$ $\mathrm{nm}$ and the electro-optical figure of merit $\mathrm{n}^{3} \mathrm{r}_{11}=(530 \pm 60) \mathrm{pm} / \mathrm{V}$ at $\lambda=1313 \mathrm{~nm}$.

Large disadvantage of DAST NLO crystals is their low stability under laser action and moisture. Absorption edge in $560 \mathrm{~nm}$ and high absorbance at $532 \mathrm{~nm}$ [6] do not allow to use DAST for second harmonic generation of $\mathrm{Nd}: Y A G$ laser.

New class of organic non-linear materials - amionopyridine-nitrophenol based co-crystals- does not have some disadvantages of DAST. In case of second order nonlinear optical applications creation of crystalline materials requires to orient donor-acceptor chromophores in non-centrosymmetric lattice [7]. To obtain non-centrosymmetric packing, heterocyclic molecules of different aminopyridines were co-crystallized with NLO chromophore 4nitrophenol (4N). Aminopyridine complexes are well-known series of compounds [8-12] with good optical properties, thermal stability [10-12], and suitability for optoelectronic devices. 4-Nitrophenol is a classic dipolar NLO chromophore, and the possibility of proton transfer of the phenolic $\mathrm{OH}$ of 4-nitrophenol to various organic bases results in increasing of its molecular hyperpolarizability $[8,13]$.

In comparison to well know NLO crystals MNA, DAST, COANP, new aminopyridine - nitrophenol complexes have advantage of absence of hygroscopicity and good optical threshold for laser power. First publication of crystal structures of some of this co-crystals was presented by Prakash et al. [8].

In the presented communication, we continue our previous studies in field of NLO crystals including DAST [14] and different NLO molecular co-crystals $[13,15,16]$. 
We report here single crystal growth process, X-ray studies, optical, thermal and SHG properties including first time non-linear second order coefficient measurements of a series of co-crystals of non-centrosymmetric complexes: 4-aminopyridine-4-nitrophenol-4-nitrophenolate, 3,4-diaminopyridine-4-nitrophenol-4-nitrophenolate, 2-amino-6methylpyridine-4-nitrophenol-4-nitrophenolate, 2,6-diaminopyridine-4-nitrophenol-4-nitrophenolate and 2aminopyridine-4-nitrophenol-4-nitrophenolate.

\section{Experimental}

Synthesis and Crystal growth. All initial reagents (Fig. 1) were obtained commercially and used without further purification. Crystals were grown by slow evaporation technique at a fixed temperature. This technique is widely used by several groups of scientists [8-12, 17-19]. We followed the synthetic procedure of co-crystals production with 4-nitrophenol that was published by Prakash et al. [8]. A ethanol solution of 4-nitrophenol was added to a ethanol solution of each N-base (4-aminopyridine; 3,4-diaminopyridine; 2-amino-6methylpyridine; 2,6diaminopyridine; 2-aminopyridine). Reagents has been taken in molar ratio 2:1.

Preparation of 4-aminopyridine-4-nitrophenol-4-nitrophenolate (4AP4N). 4-nitrophenol (0.926 g, $6.66 \mathrm{mmol})$ was dissolved in $100 \mathrm{ml}$ of ethanol; 4-aminopyridine $(0.313 \mathrm{~g}, 3.33 \mathrm{mmol})$ was dissolved in $33 \mathrm{ml}$ of ethanol and then mixed. The mixture of solutions has yellowish color.

Preparation of 3,4-diaminopyridine-4-nitrophenol-4-nitrophenolate (34DAP4N). 4-nitrophenol (0.926 g, 6.66 $\mathrm{mmol})$ was dissolved in $100 \mathrm{ml}$ of ethanol; 3,4-diaminopyridine $(0.363 \mathrm{~g}, 3.33 \mathrm{mmol})$ was dissolved in $36 \mathrm{ml}$ of ethanol and then mixed. The mixture of solutions has yellow/brown color.

Preparation of 2-amino-6-methylpyridine-4-nitrophenol-4-nitrophenolate (26MAP4N). 4-nitrophenol (0.926 g, $6.66 \mathrm{mmol})$ was dissolved in $100 \mathrm{ml}$ of ethanol; 2-amino-6-methylpyridine $(0.360 \mathrm{~g}, 3.33 \mathrm{mmol})$ was dissolved in $36 \mathrm{ml}$ of ethanol and then mixed. The mixture of solutions has yellowish color.

Preparation of 2,6-diaminopyridine-4-nitrophenol-4-nitrophenolate (26DAP4N). 4-nitrophenol (0.926 g, 6.66 $\mathrm{mmol})$ was dissolved in $100 \mathrm{ml}$ of ethanol; 2,6-diaminopyridine $(0.363 \mathrm{~g}, 3.33 \mathrm{mmol})$ was dissolved in $36 \mathrm{ml}$ of ethanol and then mixed. The mixture of solutions has yellow/brown color.

Preparation of 2-aminopyridine-4-nitrophenol-4-nitrophenolate (2AP4N). 4-nitrophenol (0.926 g, $6.66 \mathrm{mmol})$ was dissolved in $100 \mathrm{ml}$ of ethanol; 2-aminopyridine $(0.313 \mathrm{~g}, 3.33 \mathrm{mmol})$ was dissolved in $34 \mathrm{ml}$ of ethanol and then mixed. The mixture of solutions has yellow color.

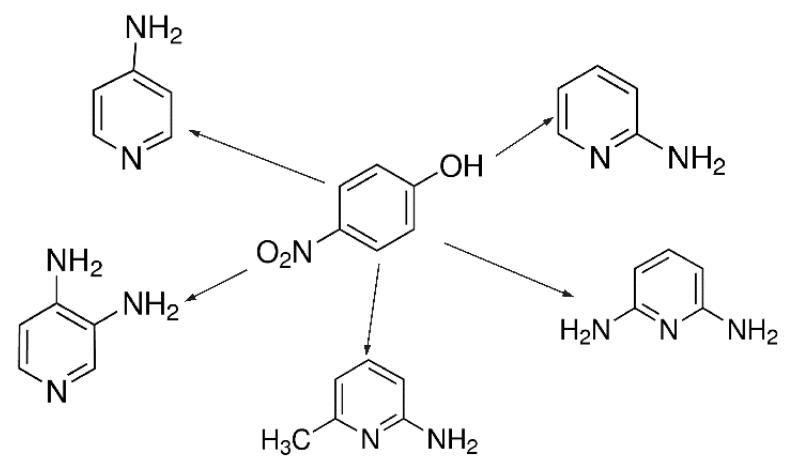

Fig. 1. Structural formulas of initial reagents

The mixture of solutions was stirred at room temperature for $1 \mathrm{~h}$ using magnetic stirrer. Continuous stirring ensured that obtained mixture of solutions is homogeneous. High purification level of synthesized compound was achieved by successive recrystallization of obtained crystals in the same solvent. The solution was filtered by funnel with quartz cells of micron size. A glass vessel with synthesized clear solution was covered with pores filter paper and placed into the thermostat at $25^{\circ} \mathrm{C}$ for slow evaporation of the ethanol. After 15-30 days, the solutions were evaporated and the crystals were grown (Fig. 2). Five co-crystals of 4-nitrophenol with different aminopyridines were grown successfully with dimensional sizes up to centimeters, namely 4AP4N, 34DAP4N, 26MAP4N, 26DAP4N, and 2AP4N. 

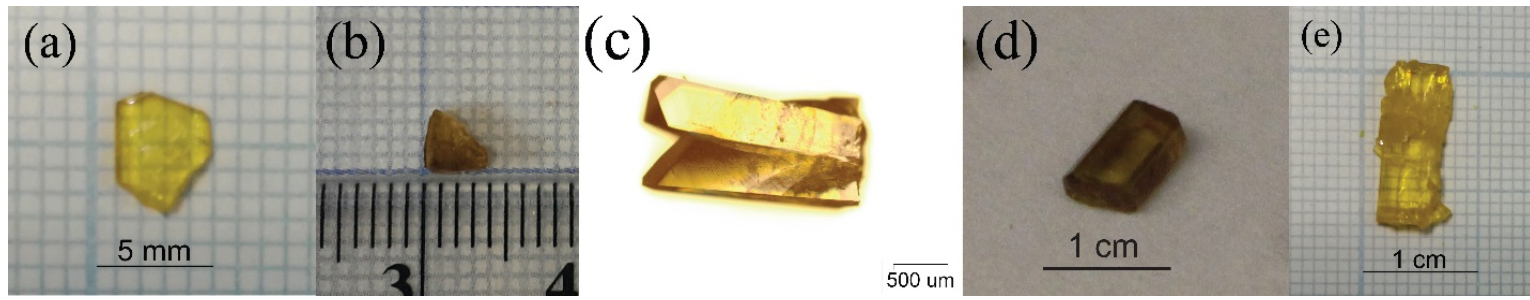

Fig. 2. Grown crystals of (a) 4AP4N; (b) 34DAP4N; (c) 26MAP4N; (d) 26DAP4N and (e) 2AP4N

$X$-ray Diffraction: Data collection for single crystal X-ray diffraction analysis of this complex was collected by Bruker-AXS SMART APEX II CCD diffractometer with monochromatized Mo K $\alpha$ radiation $(\lambda=0.71073 \AA)$ and obtained results completely corresponds to published earlier [13]. The CCDC numbers for the deposit CIF files are 886323,886324 and 886327.

UV_Vis: The UV-vis-NIR spectra of these crystals were recorded with Shimadzu UV-1800 spectrophotometer in the range 190-1100 $\mathrm{nm}$ and the recorded spectra are shown in Fig.3.

Thermal characteristics studies: The thermogravimetric and differential thermal analysis of the crystal samples was carried out using Thermo Gravimetry/Differential Thermal Analyzer(TG/DTA) HITACHI STA7200. Samples were weighted in an $\mathrm{Al}_{2} \mathrm{O}_{3}$ pans with a microprocessor driven temperature controller. TGA and DTA curves of the crystals were recorded in nitrogen atmosphere between ambient temperatures and $350^{\circ} \mathrm{C}$.

\section{Results and discussion}

\subsection{Single crystal X-Ray diffraction analysis}

Single crystal X-ray diffraction study confirms the unit cell parameters of the grown aminopyridines-4nitrophenol co-crystals as it was published earlier [13]. The acentricity is the most characteristic feature of the five reported pyridine compounds. Two of them $(4 \mathrm{AP} 4 \mathrm{~N} ; 34 \mathrm{DAP} 4 \mathrm{~N})$ crystalize in the polar $\mathrm{P} 2{ }_{1}$ space group, which allows a maximum contribution to the molecular optical nonlinearity and three compounds (26MAP4N; 2AP4N and 26DAP4N) crystallizes in acentric orthorhombic Pna2 $2_{1}$ space group. The formula units for all studied compounds are shown in table 1.

\begin{tabular}{c|ccccc}
\hline Compound & $\mathbf{4 A P 4 N}$ & 34DAP4N & 26MAP4N & 2AP4N & 26DAP4N \\
\hline $\begin{array}{c}\text { Empirical } \\
\text { Formula }\end{array}$ & $\mathrm{C}_{17} \mathrm{H}_{16} \mathrm{~N}_{4} \mathrm{O}_{6}$ & $\mathrm{C}_{17} \mathrm{H}_{17} \mathrm{~N}_{5} \mathrm{O}_{6}$ & $\mathrm{C}_{18} \mathrm{H}_{18} \mathrm{~N}_{4} \mathrm{O}_{6}$ & $\mathrm{C}_{17} \mathrm{H}_{16} \mathrm{~N}_{4} \mathrm{O}_{6}$ & $\mathrm{C}_{17} \mathrm{H}_{17} \mathrm{~N}_{5} \mathrm{O}_{6}$ \\
$\begin{array}{c}\text { Space } \\
\text { group, } \boldsymbol{Z}\end{array}$ & $\mathrm{P}_{1}, 2$ & $\mathrm{P}_{1}, 2$ & $\mathrm{Pna} 2_{1}, 4$ & $\mathrm{Pna} 2_{1}, 4$ & $\mathrm{Pna}{ }_{1}, 4$ \\
$\boldsymbol{a} / \boldsymbol{A}$ & $5.4942(4)$ & $5.5319(5)$ & $14.0366(10)$ & $13.1347(16)$ & $13.6129(14)$ \\
$\boldsymbol{b} / \boldsymbol{A}$ & $15.1735(10)$ & $15.2012(15)$ & $10.7686(7)$ & $10.912(2)$ & $10.9042(11)$ \\
$\boldsymbol{c} / \boldsymbol{A}$ & $10.4980(7)$ & $10.6928(10)$ & $11.8026(8)$ & $12.203(2)$ & $12.0323(12)$ \\
$\boldsymbol{\alpha} /^{\circ}$ & 90.0 & 90.0 & 90.0 & 90.0 & 90.0 \\
$\boldsymbol{\beta} /{ }^{\circ}$ & $101.707(1)$ & $104.581(2)$ & 90.0 & 90.0 & 90.0 \\
$\boldsymbol{\gamma}^{\circ}$ & 90.0 & 90.0 & 90.0 & 90.0 & 90.0 \\
\hline
\end{tabular}

Table 1. Crystallographic data for all studied compounds

\subsection{UV-vis studies}

For optical applications, the transparency in the visible and the near IR regions is an important parameter for materials because $1.3 \mathrm{um}$ and $1.5 \mathrm{um}$ wavelengths are widely used in telecommunications. If the crystals will be used for second harmonic generation of Nd:YAG laser, transparency in region of SH (532 nm), will be required. 
The recorded spectra of some samples (Fig.3) shows that crystals has wide transmission of above $80 \%$ up to $1100 \mathrm{~nm}$ from cutoff wavelength $(420-474 \mathrm{~nm})$. According to the recorded spectra, obtained crystals has almost the same absorption at the fundamental and harmonic wavelengths.

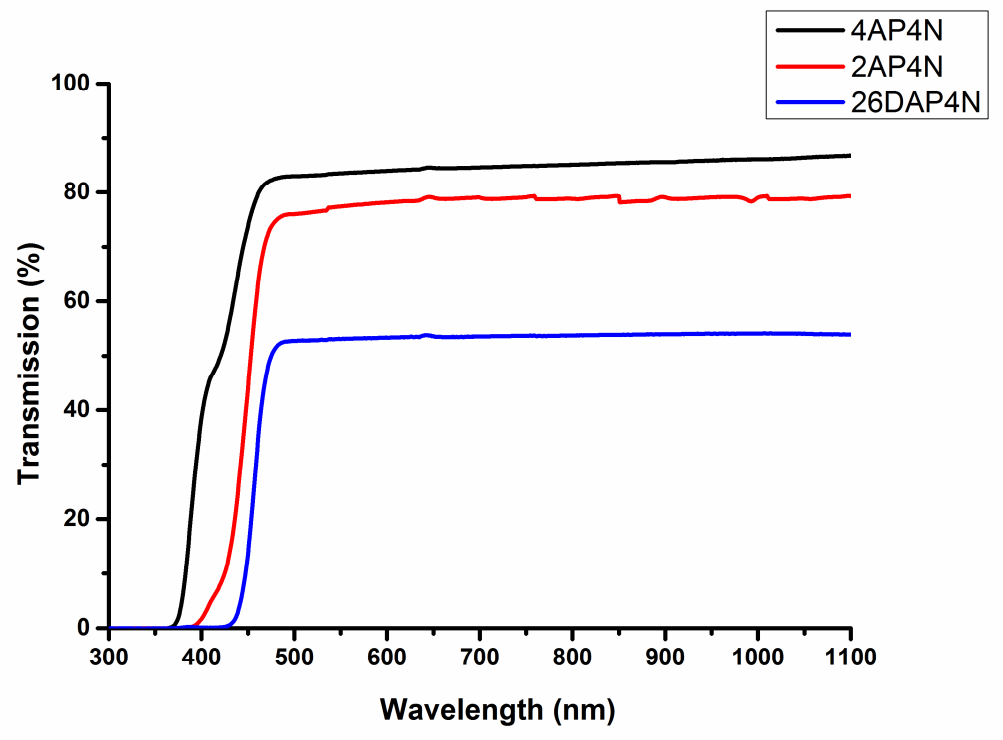

Fig. 3. Transmission spectra of studied crystals

\subsection{SHG studies}

There are several techniques to determine nonlinear optical properties of different materials [20]. In order to confirm the NLO properties, samples were subjected to comparative SHG studies [21-25]. The configuration used for evaluation of second harmonic generation is shown in Fig. 4. It consists of a pulse Nd:YAG laser as a light source. The long wavelength (RG filter) passing filters to cut off the pump lamp radiation was used. A laser beam of fundamental wavelength $1064 \mathrm{~nm}, 10 \mathrm{~ns}$ pulse width, with $10 \mathrm{~Hz}$ pulse rate was made to fall normally on the sample, mounted on the rotation stage with goniometer head. After the sample, the fundamental beam was removed by a short wavelength passing filter (BG filter), and the second harmonic signal was detected by a photodiode with sensitivity zone 420-675 $\mathrm{nm}$. A signal was displayed on a double channel oscilloscope. A reference beam of the fundamental wavelength was obtained by use of beam splitter placed ahead of the sample. This enables to be monitored the intensity of fundamental beam and triggered the SHG signal. The optical polarization is very important characteristic for SHG measurements. The optimal polarization condition was obtained when the fundamental and $\mathrm{SH}$ waves are parallel to the main axis of the crystal [26]. The vertical polarization has been chosen for SHG measurements.

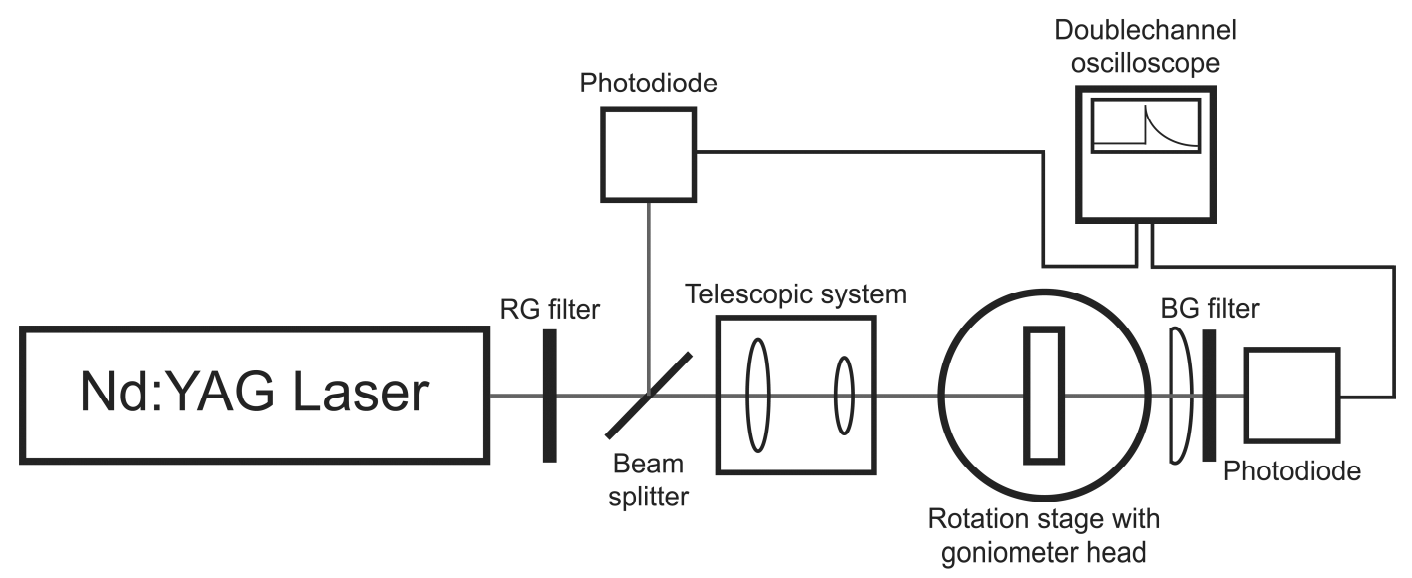

Fig. 4. Scheme for SHG measurements. 
SHG conversion efficiency of the grown single crystalline sample was measured by comparative SHG technique [23]. Since the crystal samples grow as thin plates, we did measurements using as-grown surfaces. KTP (potassium titanyl phosphate) crystal was used as reference sample in the SHG measurement. The crystals samples used for the determination of the NLO properties were rotated around an axis perpendicular to the incoming laser beam. Plot measured at rotation of one of these crystals is shown at Fig. 5.

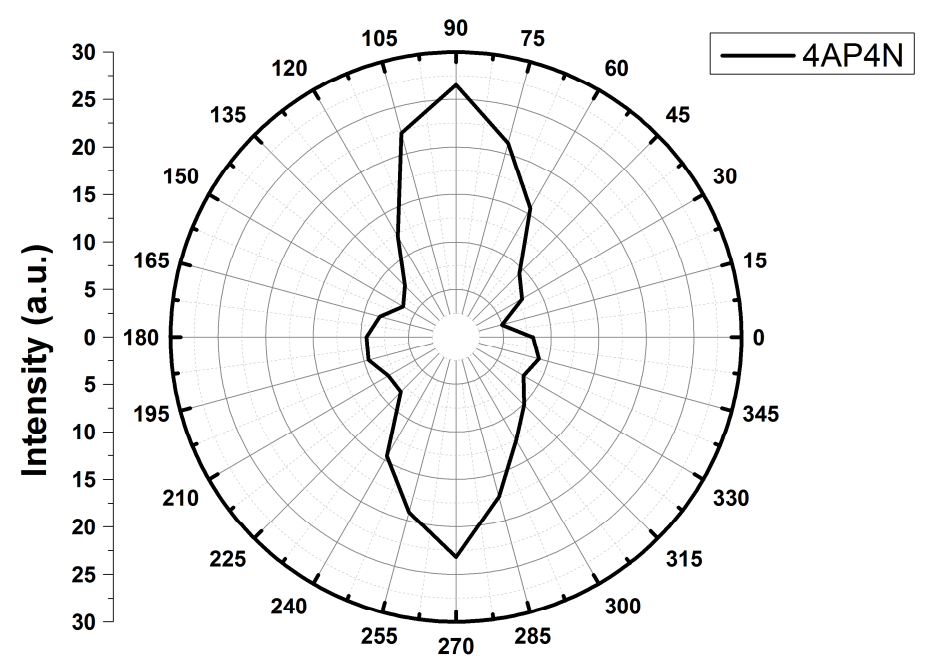

Fig. 5. Plot indicating relative intensity measured at rotation of one of the studied crystals.

To quantify the NLO response the comparison of the samples of aminopyridine co-crystals with KTP crystal was made. Taking into account the thickness of the crystals, the relative SHG efficiency of some of the compounds is found to be greater than that of KTP (which second order nonlinear optical coefficient is $13.7 \mathrm{pm} / \mathrm{V}$, according to [27]). An estimate of the magnitude of the nonlinear optical coefficient (d), at $1064 \mathrm{~nm}$, taking into account the thickness of the samples and the disordering of the crystalline regions that fall into the laser beam gives a values that are shown in table 2 , which positiones these crystals as a materials with high nonlinear optical parameters.

\begin{tabular}{cc}
\hline Crystal & $\boldsymbol{d , p m / V}$ \\
\hline 4AP4N & $0.6 \pm 0.1$ \\
34DAP4N & $13 \pm 1$ \\
26MAP4N & $6 \pm 2$ \\
26DAP4N & $21 \pm 2$ \\
2AP4N & $39 \pm 4$
\end{tabular}

Table 2. Nonlinear optical coefficients of studied crystals

If to compare measured coefficients of co-crystals with the same characteristics of MNA crystals with similar transmission spectrum we found MNA (2-methyl-4-nitroaniline) $\mathrm{d}_{33}=20 \mathrm{pm} / \mathrm{V}$ [28]; that value is similar to coefficients of co-crystals described in this paper. 


\subsection{Thermal studies}

TGA and DTA curves of the crystals studied are shown in Fig. 6-10 According to DTA analysis, grown crystals have no phase transitions between room temperature and melting point. The DTA curves show endothermic peaks with maxima at $99-172^{\circ} \mathrm{C}$ which can be recognized as the melting points of the crystals. An exothermic peak of 34DAP4N (Fig. 7) crystal sample with maximum at $287^{\circ} \mathrm{C}$ can be recognized as the full decomposition point. The second endothermic peaks of 4AP4N, 26MAP4N, 26DAP4N and 2AP4N crystals indicates full evaporation of the crystal samples, which fully correlates with the weight losses curves. According to the TGA curves, there is no weight losses between room temperature and melting points. This indicates that there is no inclusions of solvent, which was used for co-crystallization of the compounds, in the crystal lattices. The weight losses start at melting temperature and continue up to full decomposition or evaporation of the crystals samples.

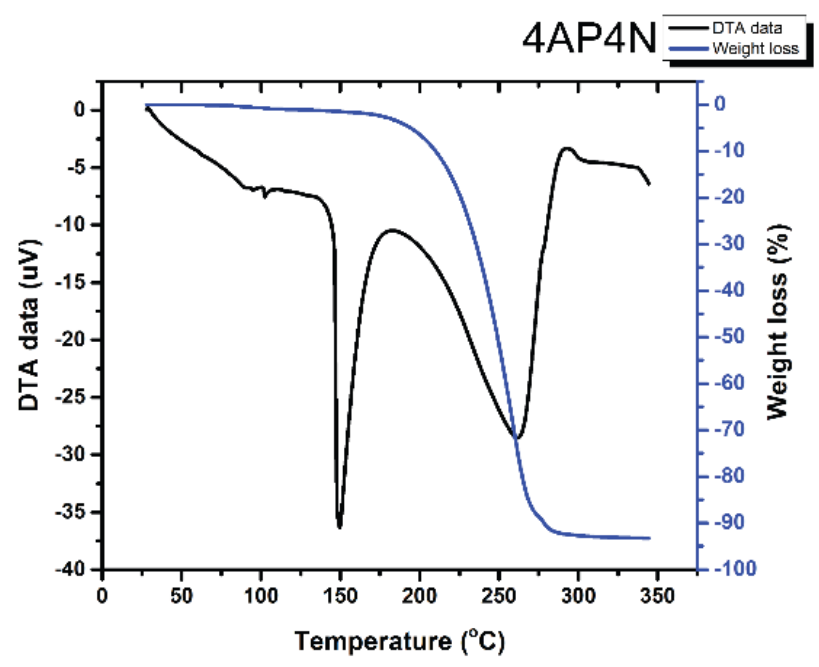

Fig. 6 TGA and DTA curves for 4AP4N crystal

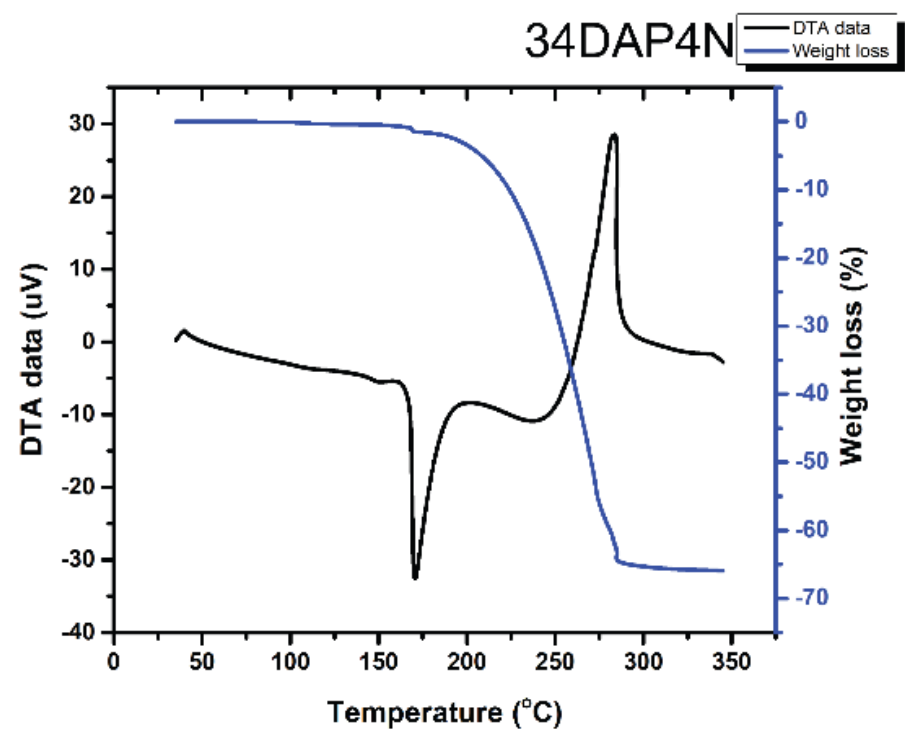

Fig. 7 TGA and DTA curves for 34DAP4N crystal 


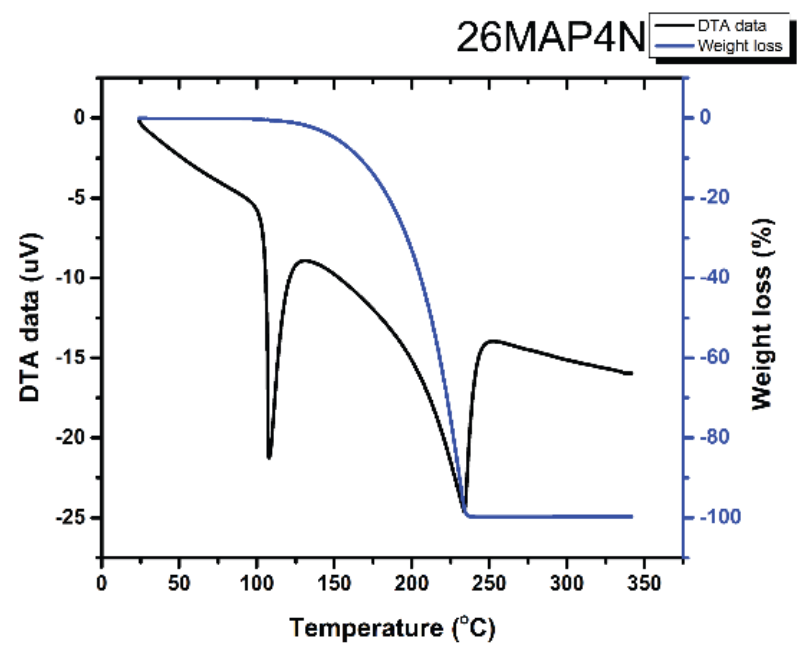

Fig. 8 TGA and DTA curves for 26MAP4N crystal

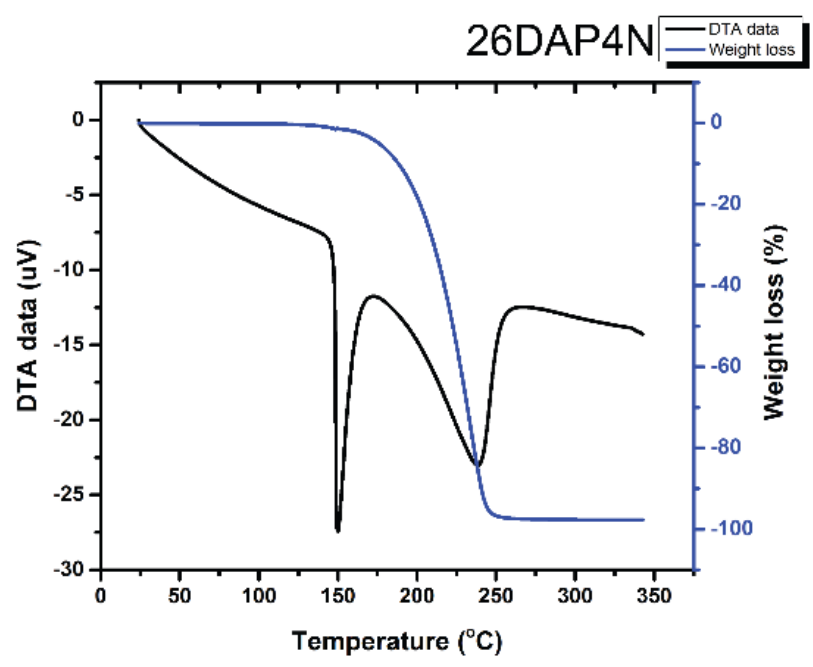

Fig. 9 TGA and DTA curves for 26DAP4N crystal

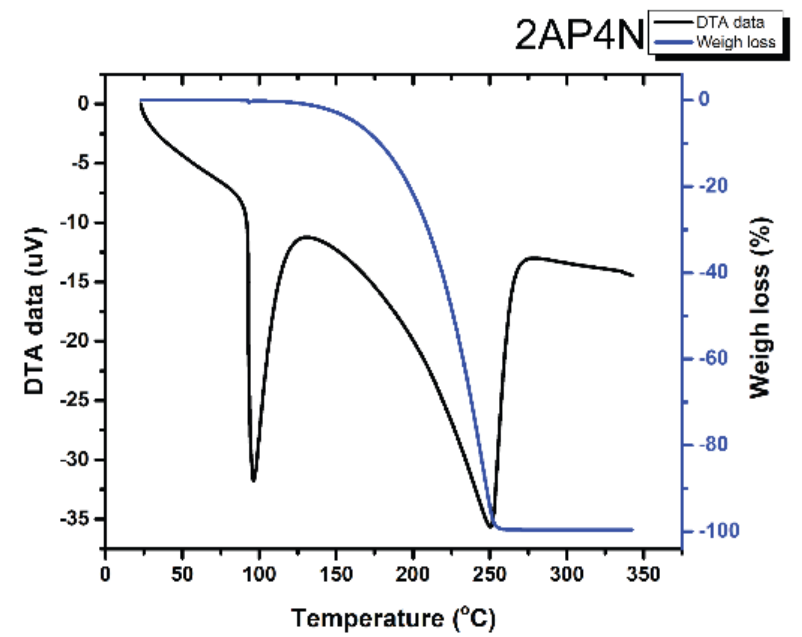

Fig. 10 TGA and DTA curves for 2AP4N crystal 


\section{Conclusion}

Good quality crystals of few aminopyridine derivatives 4-aminopyridine-4-nitrophenol-4-nitrophenolate, 3,4diaminopyridine-4-nitrophenol-4-nitrophenolate, 2-amino-6-methylpyridine-4-nitrophenol-4-nitrophenolate, 2,6diaminopyridine-4-nitrophenol-4-nitrophenolate and 2-aminopyridine-4-nitrophenol-4-nitrophenolate were grown successfully by slow evaporation technique with dimension sizes up to centimeters. Transmittance spectra show wide range of optical transmission with cut off wavelengths at 420-474 nm. Single crystal XRD studies of the grown crystals show that they are acentric with space groups $\mathrm{P} 2{ }_{1}$ and Pna2 $2_{1}$. TGA and DTA analysis shows good thermal stability and quality of the grown crystals. Second harmonic generation studies show that the grown crystals have second order nonlinear coefficient up to $39 \mathrm{pm} / \mathrm{V}$, comparable with MNA organic crystal.

\section{Acknowledgments}

Funding from the 2013-2020 Program aimed at maximizing the ITMO University's competitive advantage among the world's leading education centers 5/100. I. M. Pavlovetc, S.Draguta and T. V. Timofeeva are grateful for support via NSF DMR-0934212 (PREM) and IIA-1301346 grants.

\section{References and links.}

1. H. Figi, M. Jazbinšek, C. Hunziker, M. Koechlin, and P. Günter, Electro-optic single-crystalline organic waveguides and nanowires grown from the melt, Opt. Express, Vol. 16, Issue 15 (2008), pp. 11310-11327

2. Seth R. Marder, John E. Sohn, Galen D. Stucky, Materials for Nonlinear Optics - Chemical Represpectives, Am. Chem. Soc., Washington, DC 1991

3. S.S. Nalwa, S. Miyata, Nonlinear optics of organic molecules and polymers, CRC Press, 1997

4. Pan F., Knopfle G., Bosshard Ch., Follonier S., Spreiter R., Wong M.S., and Gunter P. Electro-optic properties of the organic salt 4-N,N-dimethylamino-4'-N'-methylstylbazolium tosylate, J. Appl. Phys. Lett. 69 (1996), 13

5. Ruiz, B., Jazbinsek, M., \& Gunter, P., Crystal Growth of DAST, J. Cryst. Growth Des. 8 (2008), 41734184

6. S. Follonier, M. Fierz, I. Biaggio, U. Meier, Ch. Bosshard, and P. Gunter, Structural, optical, and electrical properties of the organic molecular crystal 4-N,Ndimethylamino-4-N-methyl stilbazolium tosylate, J. Opt. Soc. Am. B/Vol. 19, No. 9 (2002)

7. Robert W. Boyd, Nonlinear optics. Third Edition, Academic Press, 2008

8. M. J. Prakash and T. P. Radhakrishnan, SHG Active Salts of 4-Nitrophenolate with H-Bonded Helical Formations: Structure-Directing Role of ortho-Aminopyridines, Cryst. Growth Des., 5(2) (2005), 721-725

9. J. Zhao, P. Song, Y. Cui, X. Liu, S. Sun, S. Hou and F. Ma, Effects of hydrogen bond on 2-aminopyridine and its derivatives complexes in methanol solvent, Spectrochim. Acta Mol. Biomol. Spectrosc. 131 (2014), 282-287

10. K. M. Al-Ahmary, M. M. Habeeb, E. A. Al-Solmy, Spectroscopic studies of the hydrogen bonded charge transfer complex of 2-aminopyridine with $\pi$-acceptor chloranilic acid in different polar solvents, J. of Mol. Liq. 162(3) (2011), 129-134

11. M. Shkir, B. Riscob, G. Bhagavannarayana, Synthesis, growth, structural, spectroscopic, crystalline perfection, second harmonic generation (SHG) and thermal studies of 2-aminopyridinium picrate (2APP): A new nonlinear optical material, Solid State Sci. 14 (2012), 773-776

12. P. Pandi, G. Peramaiyan, M. K. Kumar, R. M. Kumar, R. Jayavel, Synthesis, structural, optical and thermal studies of an organic nonlinear optical 4-aminopyridinium maleate single crystal, Spectrochim. Acta Mol. Biomol. Spectrosc. 88 (2012), 77-81

13. S. Draguta, M. S. Fonari, A. E. Masunov, J. Zazueta, S. Sullivan, M. Yu. Antipin, T. V. Timofeeva, New acentric materials constructed from aminopyridines and 4-nitrophenol, CrystEngComm 15 (2013), 47004710

14. J. A. Burunkova, I. Yu. Denisyuk, and M. I. Fokina, Polymer Composite Based on DAST Submicron Crystals: Technology and Properties, Mol. Cryst. Liq. Cryst. 589 (2014), 178-182

15. Sergiu Draguta Evgeniya Leonova, Maria Fokina,c Igor Denisyukc and Tatiana V. Timofeeva, N,NDiethyl-4-[(E)-(pyridin-3-yl)diazenyl] aniline, Acta Cryst. E: E69 (2013), 1280, pp 1 - 8

16. DenisyukI.,Fokina M., Pavlovetc I.,Timofeeva T.V., Fonari M., Draguta S, Comparison study of noncentrosymmetric materials from aminopyridines, in Proceeding if IEEE conference 2014 International Conference Laser Optics, LO 2014, 30 June - 4 July 2014

17. P. Srinivasan, T. Kanagasekaran, N. Vijayan, G. Bhagavannarayana, R. Gopalakrishnan and P. Ramasamy, Studies on the growth, optical, thermal and dielectric aspects of a proton transfer complex - Dimethyl amino pyridinium 4-nitrophenolate 4-nitrophenol (DMAPNP) crystals for non-linear optical applications, Opt. Mater. 30 (2007), 553-564 
18. G. AnandhaBabu, P. Ramasamy, Synthesis, crystal growth and characterization of novel semiorganic nonlinear optical crystal: Dichlorobis(l-proline)zinc(II), Mater. Chem. and Phys. 113 (2009), 727-733

19. M. Muthuraman, M. Bagieu-Beucher, R. Masse, J.-F. Nicoud and G. R. Desiraju, Sodium 4-nitrophenolate 4-nitrophenol dihydrate crystal: a new herringbone structure for quadratic nonlinear optics, J. Mater. Chem. 9 (1999), 1471-1474

20. Ch. Bosshard, J. Hulliger, M. Florsheimer, P. Gunter,Organic, Nonlinear Optical Materials, CRC Press 2001

21. U. Meier, M. Bosch, Ch. Bosshard, F. Pan and P. Gunter, Parametric interactions in the organic salt 4-N,Ndimethylamino-48-N8-methyl-stilbazolium tosylate at telecommunication wavelengths, J. Appl. Phys. 83, 7 (1998), 3486-3489

22. C. Bosshard, R. Spreiter and P. Gunter, Microscopic nonlinearities of two-component organic crystals, J. Opt. Soc. Am. B 18, 11 (2001), 1620-1626

23. W. Kaminsky, A. J. Fitzmaurice and A. M. Glazer, Measurement and calculation of second-harmonic generation in single-crystal spheres: application to d coefficients of D-mannitol, C6H14O6, J. Phys. D: Appl. Phys. 31 (1998), 767-775

24. P. D. Maker, R. W. Terhune, M. Nisenoff and C. M. Savage, Effects of dispersion and focusing on the production of optical harmonics, Phys. Rev. Let. 8 (1962), 21-23

25. J. Jerphagnonand S. K. Kurtz, Optical Nonlinear Susceptibilities: Accurate Relative Values for Quartz, Ammonium DihydrogenPhosphate, and Potassium DihydrogenPhosphate, Phys. Rev. B 1 (1970), 17391744

26. M. Sliwa, K. Nakatani, T. Asahi, P. G. Lacroix, R. B. Pansu, H. Masuhara, "Polarization and wavelength dependent nonlinear optical properties of a photo-switchable organic crystal", Chem. Phys. Lett. $437212-$ 217 (2007)

27. F. C. Zumsteg, J. D. Bierlein, and T. E. Gier, K x Rb1-x TiOPO4: A new nonlinear optical material, J. Appl. Phys. 47 (1976), 4980

28. B.F. Levine, C.G. Bethea, C.D. Thurmond, R.T. Lynch, and J.L. Bernstein, An organic crystal with and exceptionally large optical second-harmonic coefficient: 2-methyl-4-nitroaniline, J. of Appl. Phys. 50 (1979), 2523 